The Journal of Animal \& Plant Sciences, 31(4): 2021, Page: 1174-1179

ISSN (print): 1018-7081; ISSN (online): 2309-8694

\title{
EVALUATION OF PHENOLIC CONTENT AND ANTIOXIDANT ACTIVITY OF PITS AND FLESH OFDATE VAIRITIES
}

\author{
S. Khalid ${ }^{1}$, A. Ahmad ${ }^{1}$, T. Masud ${ }^{1}$ and M. J. Asad ${ }^{2}$ \\ ${ }^{1}$ Department of Food Technology, PMAS-Arid Agriculture University, Rawalpindi-Pakistan \\ ${ }^{2}$ Department of Biochemistry, Arid Agriculture University, Murree Road, Rawalpindi-Pakistan \\ Corresponding author's email: sam.butt@live.com
}

\begin{abstract}
To analyze the nutraceutical potential of different varieties of dates and phytochemical extraction capabilities of different solvents, the present study was planned to determine antioxidant activity, polyphenolic contents ofpits and flesh of three date varieties (zaidy, aseel and ajwa) were determined in different solvents (Acetone, Methanol and Ethanol) and their respective three concentrations $(70 \%, 80 \%$ and $90 \%)$. Correlation among all parameters for both parts were analyzed through Pearson correlation test. The results indicated pits as richest source for polyphenolic contents, DPPH and ABTS activity than flesh for all cultivars being highest in ajwa pits (3932.3 mg GAE/100g, $2956.2 \mathrm{mg}$ QEC/100g, 96.3\% and $86.2 \%$ respectively). In comparative analysis of solvents, $80 \%$ ethanol extracted highest antioxidant activity in all date parts. For polyphenols, different solvents have different effects on date parts so a single solvent cannot be recommended. Correlation analysis showed that phenolics have substantial positive correlation with flavonoids, DPPH and ABTS while flavonoids also revealed intensely positive relationship with DPPH and ABTS. DPPH and ABTS also exposed strongly increasing relation. In PCA analysis phenolics, flavonoids and antioxidant activity showed strong correlation in pits while showed weak relation in flesh parts. This study classifies erraticism between pits and flesh parts of date cultivars for their bioactive components which can be utilized as active ingredients against different maladies.
\end{abstract}

Key words: Phenols, DPPH, Ajwa, PCA, Pits

https://doi.org/10.36899/JAPS.2021.4.0315

Published online January 04, 2021

\section{INTRODUCTION}

Date palm (Phoenix dactylifrea) is a well renowned, oldest cultured plant in the world due to its substantial health, economic and nutritional advantages in addition to its artistic and biologicalimportance. Presence of natural antioxidants and phenolics in dates pits and flesh provide protection against different maladies like diabetes (Tahraoui et al., 2007), atherosclerosis (Abu-Elteen (2000), cancer, (Vayalil et al., 2011) and bacterial infections (Sallal et al.,1989; Al-Farsi et al., 2008). Date pits although being rich in phenolic and antioxidant content are neglected part and treated as waste product. Besides this, date varieties and their parts have hugequantity of antioxidantcontent which may differ in date varieties due to difference in their phenolic and flavonoids contents. The differences in quantity of these antioxidants depend on many factors including, extraction methods, variety and parts of date fruit (Naczk and Shahidi 2006). The extraction method is important to quantify the phenolic and antioxidant contents because different solvents with different concentrations might have effect on the quantity of these antioxidants (Santas et al., 2008). However, among all other date varieties, Ajwa date can be distinguished as it has been found to have more nutritional and health benefits as well as favorite date of Holy Prophet (PBUH) and there are some sayings of Holy Prophet
(PBUH) regarding the superiority of Ajwa among other kinds of dates. The nutritional and antioxidant activity of Ajwa flesh has been reported by several scientists (Ebtesam et al., 2011; Zhang et al., 2003) but there is no prior study on antioxidant properties of Ajwa pits. So, the study was planned to extract the phenolic contents and antioxidant activity of pit and flesh of Ajwa variety in comparison to local date varieties, in three different solvents with three different concentrations.

\section{MATERIALS AND METHODS}

Samples preparation: Ajwa date samples were purchased from renowned stores of Saudi Arabia and were recognized botanically from department of Botany PMASArid Agriculture University, whereas all other date samples were purchased from Date PlamResearch Centre Jhang, Pakistan. Fully developed fruits of uniform size, free of fungal infection and any physical injury were selected followed by their washing with distilled water and drying whereas date pits were grinded with heavy duty grinder and all samples were stored at ambient temperature until used for further analysis.

Preparation of extracts: Date flesh and pits samples were extracted separately using three solvents methanol, ethanol 
and acetone in three different concentrations $(70 \%, 80 \%$ and $90 \%$ ) for each solvent as explained in Fig. 1.

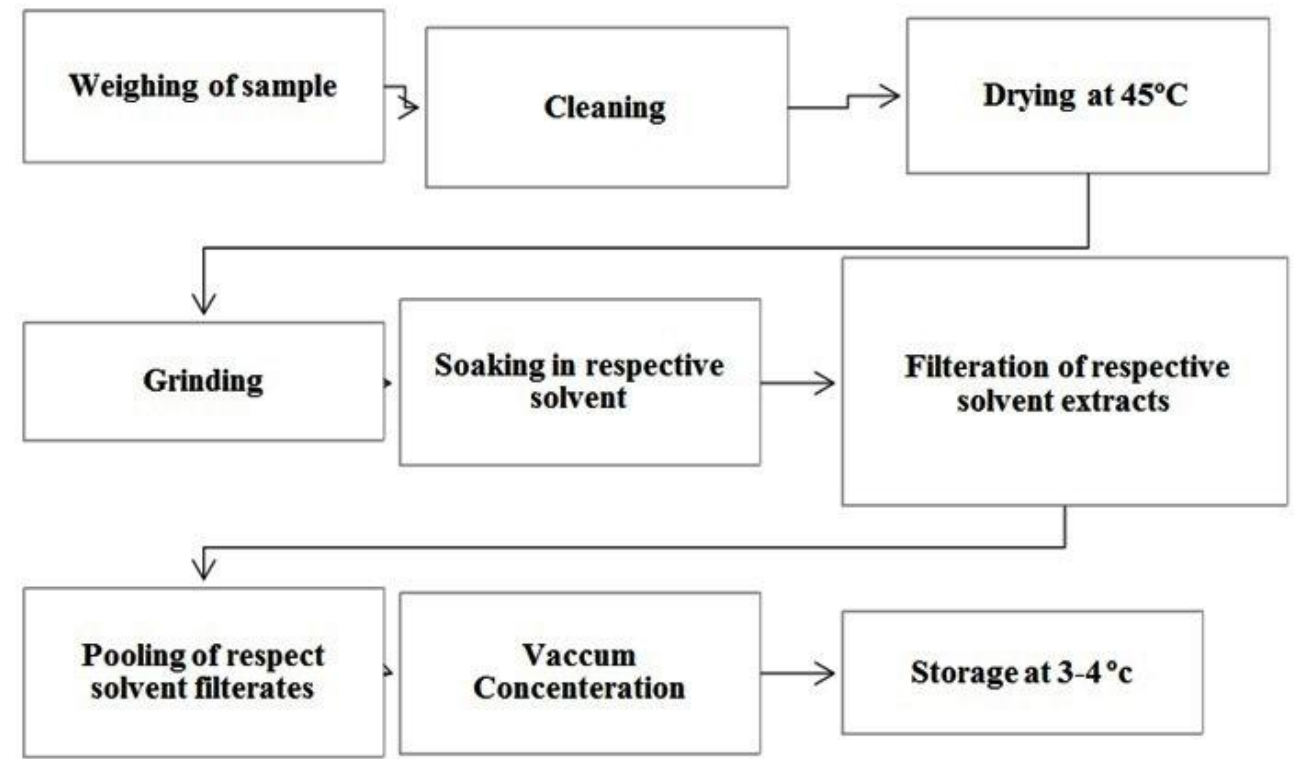

Fig. 1: Schematic diagram for extraction in different solvents

Total phenolic contents: Total phenolic contents (TPC) of both flesh and pits of all date samples were determined by Folin- Ciocalteiu reagent method of Benzie and Strain (1999). The concentration of TPC in date samples were expressed as GAE/100g (Gallic acid equivalent).

Total flavonoids contents: The TFC (total flavonoids contents) in all date samples were determined by DPPH assay according to the method of Kim et al., 2003.The concentration of total flavonoid contents in date samples was stated as $\mathrm{mg} \mathrm{QEC} / 100 \mathrm{~g}$ equivalent.

DPPH scavenging activity: DPPH solution was prepared for determination of antioxidant activity in date samples according to the method of Brand-Williams et al., (1995). Concisely, DPPH solution was prepared by dissolving $7.8 \mathrm{mg}$ DPPH in $100 \mathrm{ml}$ methanol and then from this solution $0.5 \mathrm{ml}$ was dissolved in $1 \mathrm{ml}$ of each date sample extract followed by their rest in dark room for 30 minutes at room temp. The decrease in absorption of extract mixtures at $517 \mathrm{~nm}$ was measured by using UVspectrophotometer, the reading was further used to calculate DPPH scavenging capacity by given formula:

Where:

$$
\mathrm{DPPH}=\mathrm{A}^{0}-\mathrm{A} 1 / \mathrm{A}^{0} \times 100 \text {, }
$$

$\mathrm{A} 0=$ control absorbance and $\mathrm{A} 1=$ sample absorbance

ABTS scavenging activity: ABTS solution was prepared by adopting method of Arnao et al. (2001). Briefly, $7 \mathrm{mM}$ ABTS solution was countered with $2.46 \mathrm{mM} \mathrm{K}_{2} \mathrm{SO}_{4}$ and whole solution was left in a dark room overnight. After that, the mixture was dissolved with $5 \mathrm{mM}$ saline phosphate buffer until 0.70 absorbance was achieved at
$730 \mathrm{~nm}$ followed by reaction of $4 \mathrm{ml}$ of this diluted ABTS solution with $10 \mu \mathrm{l}$ extracts samples after 30 minutes. The ABTS scavenging activity for all samples was expressed as:

$$
\text { ABTS Scavenging }(\%)=\frac{\mathrm{AO}-\mathrm{A}}{\mathrm{AO}} \times 100
$$

The extract of date parts studied with best profile of phytochemicals and their antioxidant activities will be further studied for their efficacy against induced hypercholesterolemia in animal models.

Statistical Analysis: To differentiate type of solvent and concentration of solvent analysis of variance technique was used, while to reduce the number of factors like part of date, variety of date, type and concentration of solvent Principal component analysis was employed, correlation and Scatterplot analysis were used to observe relationships within various parameters. For all statistical analysis R studio and Minitab version 16 software were used.

\section{RESULTS AND DISCUSSION}

Total phenolic contents: Phenolic contents of flesh and pit of different date varieties fluctuates widely in each type of solvent concentration. Solvent concentration, part of date (flesh or pits) and interaction between solvent concentration and part have a significant effect on TPC, where as all main effects and different interactions were non-significant $(\mathrm{p}>0.05)$. Flesh partsof dates have significantly lower total phenolic contents as compared to pits part(Fig.2).However, within flesh parts total phenolic contents of ajwa flesh ranged from $483.8 \mathrm{mg}$ GAE/ $100 \mathrm{~g}$ ( $80 \%$ ethanol) to $424.1 \mathrm{mg}$ GAE/ $100 \mathrm{~g}$ (70\% methanol) 
while for aseel flesh it ranged between 291.3 mgGAE/100g (90\%ethanol) to $220.3 \mathrm{mg} \mathrm{GAE} / 100 \mathrm{~g}$ (70\% acetone) whereas for zaidy flesh the values vary from $322.8 \mathrm{mg}$ GAE $/ 100 \mathrm{~g}(90 \%$ methanol $)$ to $200.3 \mathrm{mg}$ GAE $/ 100 \mathrm{~g}(70 \%$ methanol). Similarly, for pits, Ajwa pits have range of phenolic contents between $3932.3 \mathrm{mg}$ $\mathrm{GAE} / 100 \mathrm{~g}(90 \%$ ethanol $) \quad$ to $\quad 3154.7 \quad \mathrm{mg}$ GAE $/ 100 \mathrm{~g}(70 \%$ ethanol) while values vary from 2655.4 $\mathrm{mg}$ GAE $/ 100 \mathrm{~g}(90 \%$ ethanol) to $2603.7 \mathrm{mg}$ GAE $/ 100 \mathrm{~g}$ (70\% acetone) in aseel pits. Similarly, zaidy pits have a range of total phenols from $2432.2 \mathrm{mg}$ $\mathrm{GAE} / 100 \mathrm{~g}(90 \%$ ethanol $)$ to $2333.5 \quad \mathrm{mg}$ GAE $/ 100 \mathrm{~g}(70 \%$ ethanol). Similarly, within varieties for both parts of dates (flesh and pits) same trend were observed for TPC viz; ajwa>aseel>zaidy except for $70 \%$ methanolic extracts of flesh part where ajwa flesh have highest amount of TPC followed by zaidy and then aseel flesh. The variability in results of total phenolic contents is due to many factors including; parts and variety of plant, type of solvents used, polarity of solvent and extraction method (Devshony et al. 1992). Different concentrations of solvents are being used for extraction of phenolic contents from plants and their different parts such as fruit, seeds, leaves and vegetables (Pérez-Jiménez and Saura-calixto 2006). Variable trend for TPC extraction in different solvent and their concentrations is evident. Interactive effect of solvent concentration and parts was significant showing that in some parts TPC can be extracted using $90 \%$ ethanol while in other parts can be extracted through $80 \%$ ethanol, $70 \%$ ethanol and $90 \%$ acetone. Results suggest that solvent polarity plays a basic role in enhancing phenolic solubility (Naczk and Shahidi 2006) and water-organic solvent mixtures are more effective towards the extraction of total phenolic contents. Therefore, it is difficult to develop a standard extraction procedure that will be appropriate for extraction of phenolic contents of all plants and their parts (Naczk and Shahidi 2006). Furthermore, results suggest that pits have remarkable potential for polyphenols than flesh so they can be a better source of phenolic contents than flesh parts of date cultivars irrespective of variety and solvent used. Differences in flesh and pits quantities might be due to their low moisture and sugar contents however, due to their higher potential of phenols their wastage should be minimized (Ebtesam et al., 2011Khanaviet al., 2009. The results further suggest more work on isolation of these phenols derived from date parts so that synthetic phenols can be replaced in foods.

Total flavonoid contents: Flesh and pits of date cultivars also revealed extensive differences for flavonoids contents in all varieties (Fig. 3). Solvents with different concentrations, solvent and variety type, different parts of date, interaction between concentration-part-solvent, concentration-part- variety, solvent-variety have significant effects on TFC while effect of solvents on parts and all other factors have non-significant effect on flavonoids. In general, flesh parts have lower amounts of flavonoids than pits. In flesh parts, ajwa flesh have flavonoid contents in range of 126.3 (70\% methanol) to $386.3 \mathrm{mg}$ QEC/100g ( $80 \%$ acetone) while zaidy has a range of $261.3(80 \%$ acetone) to $347.1 \mathrm{mg} \mathrm{QEC/100g} \mathrm{(90 \%}$ methanol) whereas aseel flesh has flavonoid contents varying from $179.5(90 \%$ methanol $)$ to $324.2 \mathrm{mg}$ QEC/100g (80\% acetone). Similarly, Ajwa pits have a range of flavonoids from $1897.4 \mathrm{mg}$ QEC/100g $(70 \%$

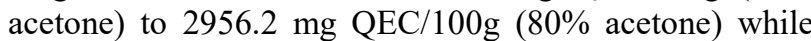
flavonoid contents of aseel pits varies from $1153.2 \mathrm{mg}$ QEC/100g (80\% methanol) to $2143.2 \mathrm{mg}$ QEC/100g (80\% acetone) whereas for zaidy pits flavonoids have a range between $1013.6 \mathrm{mg}$ QEC/100g (90\% methanol) - 1962.3 $\mathrm{mg}$ QEC/100g (80\%actone). Trend of TFC in varieties within both parts was same as for phenolic i-e ajwa $>$ aseel $>$ zaidy. Significant effect of solvent and its different concentrations on TFC suggests that flavonoids are dependent on the type of solvents used, polarity of solvent and extraction method. Water -solvent polarityplays a basic role in enhancing flavonoids solubility (Naczk and Shahidi 2006). Results further suggest that $80 \%$ acetone was the best solvent to extract flavonoids from both parts of all date cultivars recommending it as best solvent to extract flavonoid contents which might be due to its higher capacity to dissolve polar flavonoids. In general, pits of date varieties are better source of flavonoids than flesh. The variation in flavonoid contents of flesh and pits might be due to their genetic makeup, solvent used and solvent concentration suggesting more studies on date pits to explore their flavonoids and for making possible their use in food industry to minimize their wastage. Further studies are also recommended for extraction of non-flavonoids contents from date parts and cultivars.

Total antioxidant activity: Different methods are employed for evaluation of antioxidant activity in fruits, of which ABTS and DPPH are most common. These methods have different reaction procedures that elucidate the antioxidant activity of fruit components. For that purpose, it is suggested to use at least two methods to provide reliable antioxidant activity of samples. In this study, antioxidant activity of date flesh and pits were evaluated by two methods viz. DPPH and ABTS. Moreover, antioxidant activity is affected by solvent type, concentration, varieties and their different parts.For DPPH assay, solvent concentrations, parts and interaction of solvent- part- concentration have significant effects on antioxidant activity of date parts while non-significant effect of different varieties and all other interactions was observed in all date samples. Generally, date pits have higher antioxidant activity than flesh parts for all cultivars in all solvent concentrations. In flesh parts, ajwa flesh exhibited highest antioxidant activity in the range of 77.2 
$\%$ ( $80 \%$ ethanol) to $62.7 \%$ ( $70 \%$ methanol) while zaidy flesh showed least quantity of antioxidants ranging between $58.2 \%$ (80\% ethanol) to $39.8 \%$ ( $70 \%$ ethanol) in all solvent's extracts. Similarly, in pits ajwa pits exhibited maximum antioxidant activity ranging between $96.3 \%$ ( $80 \%$ ethanol) to $67.3 \%(70 \%$ acetone) while zaidy pits has lowest antioxidant activity varying from $67.1 \%(70 \%$ ethanol) to $47.6 \%$ (90\% methanol) in different solvents.

Similarly, in ABTS assay solvent, variety, concentrations of solvent, solvent and variety interaction have significant effect on antioxidant activity whereas all other interactions have non-significant effect. Normally, date pits significantly showed more antioxidant activity in comparison to date flesh of all varieties. Within parts, ajwa flesh exposed maximum ABTS scavenging activity ranging between $73.5 \%$ (80\% ethanol) to $54.2 \%$ whereas zaidy flesh have minimum ABTS scavenging activity varying from $49.5 \%$ (70 to $38.6 \%$. Similarly, for pits parts ajwa pits have highest amount of antioxidant activity in range of $86.2 \%$ to $69.3 \%$ followed by zaidy pits which have antioxidant activity varying from $65.9 \%$ to $54.2 \%$. Results suggest that the antioxidant activity of different extracts has strong association with different concentrations of solvent used, mainly due to different scavenging capacity of compounds with different polarities (Pérez-Jiménez and Saura-calixto 2006).
Therefore, it can be suggested that antioxidants are more dissolved in polar solvents and among them ethanol and its water concentrations $(80 \%$ and $70 \%)$ are most effective solvent for extraction of date antioxidants due to a wide range of compounds that it can dissolve. Differences among pits and flesh recommend pits to be a great source for antioxidants which might be due to their higher phenolic and flavonoid contents. However, further studies are recommended for isolation of these antioxidants.

Correlation analysis: Phenolic contents have significantly higher positive correlation with flavonoids (0.96), DPPH (0.63) and ABTS (0.75). Similarly, strongly positive correlation was observed between flavonoids and DPPH (0.61) and ABTS (0.74) while DPPH and ABTS $(0.89)$ also revealed strongly positive increasing relationship as evident in Fig. 2.Irrespective of date parts and solvents, histogram shows that most of date cultivars have phenolic contents ranged between $200-1000 \mathrm{mg}$ GAE/100g, flavonoids between 300-1200 mg QEC/100 g and DPPH and ABTS contents between 55-65\% (Fig.2). Results suggest that phenolic and flavonoid contents have strong effect on antioxidant activity of date fruit. So above studied date varieties can be classified on single attribute from above stated parameters while studying their antioxidant properties.

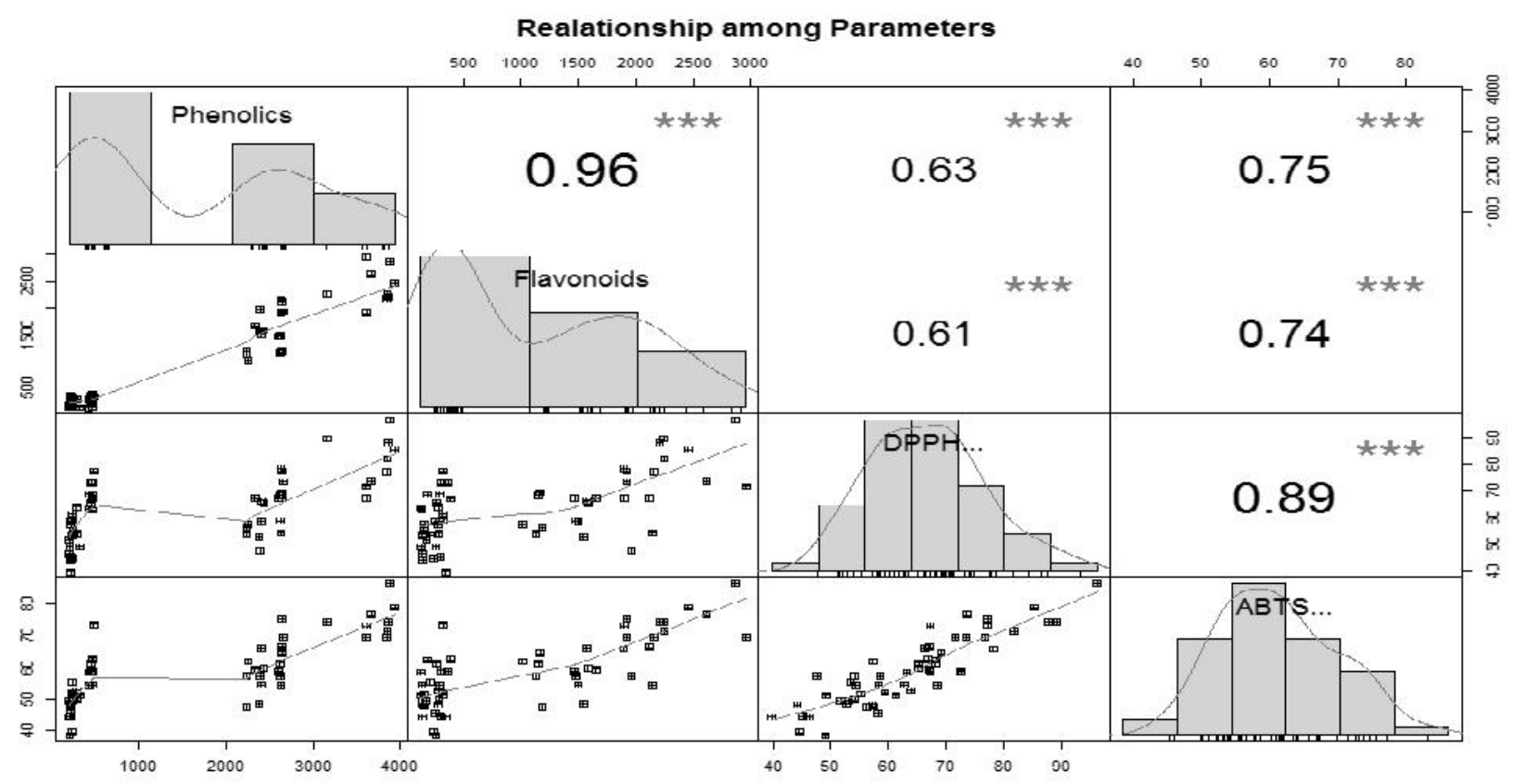

Fig.2: Relationship between Phenolics, Flavonoids and antioxidants.

Principle component analysis: New plant sources for natural antioxidant are interesting to replace synthetic antioxidants. Many literature reports designate studies of antioxidants from date palm and their parts. Hence, it is important if we classify date parts based on their total phenolic, total flavonoid contents and antioxidant activity.
From above results, it is investigated that pits have higher amount of TPC, TFC while for antioxidant activity less variation between both parts were observed. To understand more about variables and variation between date parts PCA was applied based on their antioxidant, phenolic and flavonoids contents. PCA shows variability 
among date parts in two dimensions. The two principle components accounted for $82.3 \%$ and $14.5 \%$ of variability for data. The leading PC1 has strong positive correlation for phenolic, flavonoid and antioxidant contents of pits while less strong positive correlation of PC 2 was for antioxidant, flavonoids and phenolic contents of flesh part. Two classes were grouped and used to generate plant parts image on bi-plot (Fig.7.). Pits and flesh are clearly separated from each other based on their phenolic, flavonoid and antioxidant contents. This technique provides a useful tool for understanding much more about the clarification of results from research studyand their effect of date parts on them. Exploration of phenolics, flavonoids and antioxidants in each part can assist in the classification of date cultivars and parts.

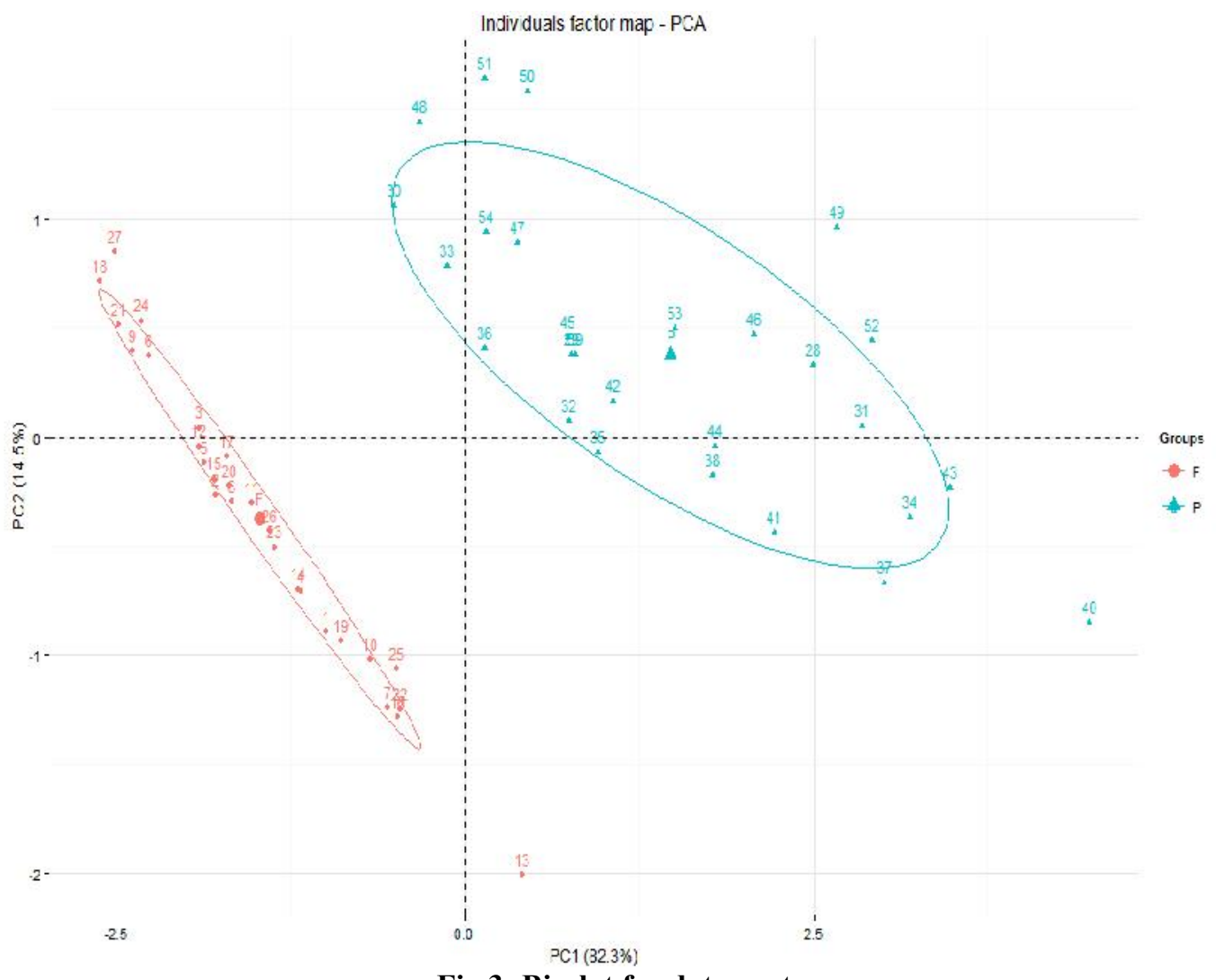

Fig.3: Bi-plot for date parts

Conclusion: Comparison analysis between date parts of above studied three date varieties showed higher quantities of total phenolic, total flavonoids and antioxidant contents in date parts whereas ajwa pits were found ironicsource of all above parameters in all date varieties. Whereas, in comparative analysis of solvent and their concentrations, it is concluded that solvent type has non-significant effect on phenolic, flavonoid contents and antioxidant activity while solvent concentration has significant effect on all parameters but no signal solvent can be suggested for extraction of phenolic and flavonoidshowever, 70\% and $80 \%$ concentration of all solvent was found good for DPPH and ABTS assay in both parts of all date varieties. In correlation analysis, phenolic contents have significant positive correlation with flavonoids, DPPH and ABTS. Similarly, strongly positive correlation was also observed between flavonoids and DPPH and ABTS while DPPH and ABTS also revealed strongly positive increasing relationship. Based on PCA analysis, the data group was clearly divided into two subsets; pits were separated from flesh parts based on their phenolic contents, flavonoid contents and antioxidant activity which will be helpful in classification of date parts and cultivars. Comparative analysis between date fruit parts in different solvent extracts will be helpful when studying the beneficial properties of date cultivars as medicinal plant to be used for natural antioxidants. Furthermore, this work will givesentience to publicand consumers concerning the antioxidant effects ofall date pits especially ajwa pits so that diminution of date pits could be lessened. Elevatedphenolic contents and antioxidantactivity in date pits than flesh specifically ajwa date stress their consumption in food and pharmaceuticalmanufacturing as functional and disease protective ingredient in food products and supplements. 
Acknowledgement: Authors are highly acknowledged to Higher Education Commission for financial support throughout the project.

\section{REFERENCES}

Abu-Elteen, K.H. (2000). Effects of date extract on adhesion of Candida species to human buccal epithelial cells in vitro. J. Oral Pathol.Med. 29:200-205.

Al-Farsi, M. A., \& C. Y. Lee. (2008). Nutritional and functional properties of dates: a review. Critical Reviews in Food Science and Nutrition. 48: 877887.

Arnao, M. B., A. Cano and M. Acosta. (2001). The hydrophilic and lipophilic contribution to total antioxidant activity. J. Food Chem.73:239-244.

Benzie, I. F. F. \& J. J. Strain. (1999). Ferric reducing/antioxidant power assay: Direct measure of total antioxidant activity of biological fluids and modified version for simultaneous measurement of total antioxidant power and ascorbic acid concentration. Methods. Enzymol. 299:15-27.

Brand-Williams, W. M.E. Cuvelier and C. Berset. (1995). Use of free radical method to evaluate antioxidant activity. Lebensm. Wiss. Technol. 28: 25-30.

Devshony, S., A. Eteshola and A. Shani. (1992). Characterization and some potential application of date palm (phoenix dactylifera 1.) seeds and seed oil. J. American. Oil Chem. Soci. 69: 595-597.

Ebtesam, S., M.S. Tawakif, H.M. Abu-Taroush. (2011). Phenolic contents and antioxidant activity of various date palm (phoenix dactylifera 1.) fruits from Saudi Arabia. 2(10):1134-1141.

Kim, J. K., S. Lee, J. S. Choi Suh, H. Y. Chung, Y. O. Song, W. C. Choi. 2003. The first total synthesis of 2, 3, 6-tribromo-4, 5-dihydroxybenzyl methyl ether (TDB) and its antioxidant activity. Bull Korean Chem. Soc., 23: 661-662.

Khanavi, M., Z. Saghari, A. Mohammadirad, R. Khademi, A. Hadjiakhoondi and M. Abdollahi. (2009). Comparison of antioxidant activity and total phenols of some date varieties. Daru. 17: 104-107.

Naczk, M., and F. Shahidi. (2006). Phenolics in cereals, fruits and vegetables: Occurrence, extraction and analysis. J. Pharm. Biomed. Anal, 41; 1523-1542.

Perez-jimenez, j. and F. Saura-calixto. (2006). Antioxidant capacity of dietary polyphenols determined by abts assay: a kinetic expression of the results. Int. J. Food sci. Technol., 43: 185- 191.

Sallal, A.K., A. Ashkenani. (1989). Effect of date extract on growth and spore germination of Bacillus subtilis. Microbios. 59:203-210.

Santas, J. R. Carbó., M. H. Gordon and M. P. Almajano. (2008). Comparison of the antioxidant activity of two Spanish onion varieties. Food Chem. 107; 1210-1216.

Tahraoui, A., J. El-Hilaly, Z. H. Israili, B.Lyoussi. (2007). Ethnopharmacological survey of plants used in the traditional treatment of hypertension and diabetes in south-eastern Morocco (Errachidia province). J. Ethnopharmacol. 110:105-117.

Vayalil, P. K. (2011). Date fruits (Phoenix DactyliferaLinn): An emerging medicinal food. Crit. Rev. Food. Sci. Nutr. 52: 249-271.

Zhang, C.-R., S. A. Aldosari, P. S. Vidyasagar, P. Shukla and M. G. Nair. 2003. Health-benefits of date fruits produced in Saudi Arabia based on in vitro antioxidant, anti-inflammatory and human tumor cell proliferation inhibitory assays. J. Saudi Society Agri. Sci., 25: 584-592. 